\title{
Temporally programmed polymer - solvent interactions using a chemical reaction network
}

Benjamin Klemm ${ }^{a}$, Reece W. Lewis ${ }^{a}$, Irene Piergentilia , and Rienk Eelkema ${ }^{a}{ }^{*}$

a Delft University of Technology, Department of Chemical Engineering, Van der Maasweg 9, $2629 \mathrm{HZ}$ Delft, The Netherlands.

*Correspondence to: R.Eelkema@tudelft.nl 


\begin{abstract}
Chemical reaction networks (CRNs) that operate under constant influx of energy enable artificial materials to autonomously respond to their environment by activation and deactivation of intermolecular interactions. Generally, their activation can be driven by various energy sources, yet their deactivation to non-interacting building blocks remains largely limited to hydrolysis and internal $\mathrm{pH}$ change. To achieve control over deactivation, we developed a new CRN that enables reversible formation of positive charges on a tertiary amine substrate, which are removed using nucleophilic signals that control the deactivation kinetics. Incorporation of the CRN in a polymer material leads to a temporally programmed transition from collapsed and hydrophobic to solvated, hydrophilic polymer chains by controlling polymer-solvent interactions. Depending on the layout of the CRN, we can create stimuli-responsive or autonomously responding materials. This concept will not only offer new opportunities in molecular cargo delivery but also pave the way for next-generation interactive materials.
\end{abstract}




\section{Introduction}

Interactive materials, which are able to adapt and to interact with their surroundings by responding to events taking place in their environment ${ }^{1}$, will find many applications ranging from regenerative medicine, optoelectronics to nanomachines. ${ }^{2-4}$ Response to environmental cues through controlled material growth and decay is a unique property, which enables nature to perform complex functions, for example signal transduction, cell-division and intracellular transport. ${ }^{5,6}$ Here, the conversion of chemical fuels such as adenosine triphosphate (ATP) are used to temporally control out-of-equilibrium assembly into supramolecular structures. ${ }^{7}$

Synthetic analogues of these active materials can be designed by coupling the activation of non-interacting building blocks to fuel-driven chemical reaction networks (CRNs). ${ }^{8}$ Crucially, the availability of chemical fuel sustains the active material and depletion of the fuel causes the material to disassemble back to its precursor. ${ }^{9}$ Besides strategies that involve activation of the surroundings, such as a transient $\mathrm{pH}$ change ${ }^{10-13}$, a widely applied strategy is the direct activation of building blocks by a chemical fuel. ${ }^{9}$ In such systems, the chemical fuel reacts with an inactive building block, converting it to an activated intermediate (activation). A second reaction subsequently converts the intermediate to its inactive precursor state by spontaneously forming a waste product (deactivation). ${ }^{14}$ Autonomously dynamic materials made using fuel-driven out-of-equilibrium CRNs include microgels ${ }^{15}$, supramolecular gels ${ }^{11}$, nanoparticles ${ }^{16}$ and vesicles ${ }^{17}$. These materials use a wide variety of material activation processes including different chemical fuels, light or ultrasound to name a few. ${ }^{18-20}$ In contrast, the deactivation mechanism in chemically fuelled non-equilibrium CRNs relies frequently on hydrolysis ${ }^{9,15,16,21-23}$ or internal $\mathrm{pH}$ change $\mathrm{e}^{10-13}$ and only a few non-enzymatic alternatives have been investigated ${ }^{24-26}$. Regulation of the deactivation reaction in CRNs remains a key issue in out-of-equilibrium systems. ${ }^{27}$

In an effort to expand the current possibilities and to achieve control over the deactivation reaction, we envisioned CRNs based on nucleophilic substitution by using allylic electrophiles as chemical fuel. Such substrates are highly versatile with a wide range of reactivities and 
structures $^{28,29}$. In particular, the wide range of nucleophilic reactivities and their prevalence in nature opens the door to nucleophilic control over the deactivation step, enabling highly tuneable and versatile CRNs. In this work, we introduce a new strategy toward interactive synthetic materials. Specifically, the materials undergo a temporally programmed transition from collapsed, hydrophobic polymer chains to solvated, hydrophilic polymer domains by controlling polymer-solvent interactions. We can achieve stimuli-responsive or autonomous material changes by controlling these interactions using our CRN strategy to reversibly form positive charges along a polymer backbone at physiological $\mathrm{pH}$. We show the adaptability of this strategy by applying it to block copolymer micelles as well as macromolecular hydrogel superstructures. 


\section{Results and Discussion}

The CRN central to this work is based on the allylic substitution of electron deficient allyl acetates with tertiary nitrogen nucleophiles. We observed that in this reaction, a metastable, positively charged quaternary nitrogen adduct is formed ${ }^{30,31}$. From there, we discovered that this ammonium-allyl adduct (the activated intermediate) is stable in buffered aqueous solution at neutral $\mathrm{pH}$ and room temperature until undergoing nucleophilic attack. Competing $\mathrm{S}$ or $\mathrm{N}$ terminal nucleophiles can perform a second allylic substitution on the activated intermediate ${ }^{32,33}$, which reverses the quaternary nitrogen to the neutral amine adduct, forming the allylic reaction product (waste) and thus completing the reaction cycle (Figure 1a). In this cycle, the allyl substrate diethyl(a-acetoxymethyl) vinylphosphonate (DVP) ${ }^{34}$ acts as a fuel, enabling a reversible switch between charge states of the nitrogen centre. We are able to manipulate the $\mathrm{CRN}$ and the subsequent material response by delicate design of the allylation reaction (activation) and its successive substitution reaction in the presence of nucleophiles (deactivation). Highly nucleophilic thiols in water ${ }^{35,36}$, such as 2-mercaptoethanol (SH-3, Figure 1b), react first with the activated intermediate before attacking the fuel itself. This allows for signal-controlled cycling between 'charged' and 'uncharged' species, referred to as signalinduced cycling (Figure 1a-right). We studied two common tertiary amines (Figure 1b) in a small molecule CRN: DABCO (t-Am-1) and pyridine (t-Am-2) and evaluated their behaviour in the CRN. Furthermore, we investigated the potential of chemically fuelled, out-of-equilibrium systems, where competition of (de)activation reactions leads to autonomous cycling (Figure 1a-right). Here, we sought for weak nucleophiles, which were not reactive with the fuel. Amino acids such as threonine $\left(\mathrm{NH}_{2}-4\right.$, Figure $\left.1 \mathrm{~b}\right)$ are prime candidates, due to their weak nucleophilicity (nucleophilicity index $(N)=12.69$ in water ${ }^{37}$ ). The primary amine $p K_{a}$ of threonine is 9.1 meaning that at neutral $\mathrm{pH}$, it is mostly present as the protonated amine species $(>97 \%) .{ }^{38}$ Under optimised conditions, this allows for an initial accumulation of the charged activated intermediate, which is later reversed by the delayed substitution from excess $\mathrm{NH}_{2}-4$ to form the uncharged waste products (DVP-N + DVP-2-N, Figure $1 \mathrm{~b}$ ). 
To bridge this concept towards synthetic materials, we synthesised block and statistical copolymers of N,N-dimethylacrylamide and 4-vinylpyridine (P1 and P2, Figure 1C-left) by a two-step reversible addition fragmentation chain-transfer (RAFT) process. ${ }^{39}$ The $\mathrm{pK}_{\mathrm{a}}$ of poly(4vinylpyridine) is $5.0 \pm 0.3^{40}$ meaning that at neutral $\mathrm{pH}$ the free base species is dominant, making it an excellent candidate for reversible charge formation in neutral $\mathrm{pH}$ buffered aqueous systems. With those polymers in hand, we prepared two types of materials: (1) supramolecular micellar dispersions and (2) macromolecular polymer hydrogels (Figure 1cright). Fuelling these materials with DVP, we anticipated polymer dissolution in micellar dispersions by induced hydrophilization and polymeric network expansion within hydrogels by osmotic pressure forced water intake. The subsequent decay of the intermediate species will convert the positive charge along the polymer, and in turn lead to micellar re-formation and hydrogel contraction due to the loss of charges and osmotic pressure ${ }^{41}$ (Figure 1d). 
a

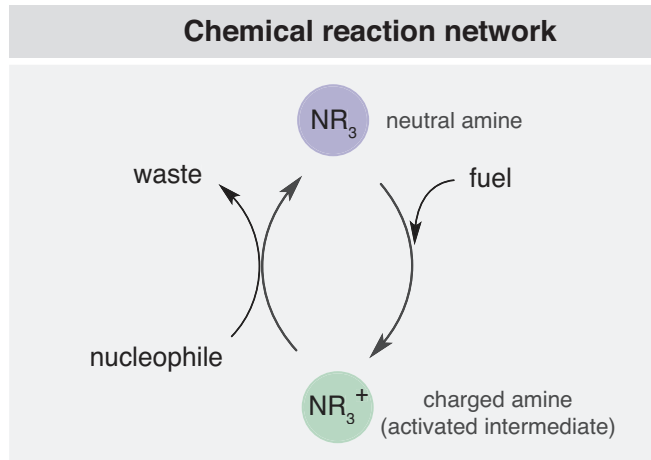

b

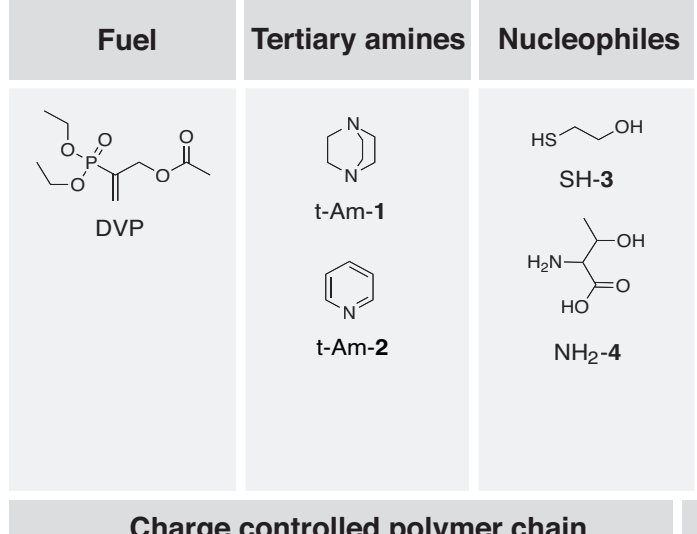

Signal - induced vs. Autonomous cycle strategy

Signal-induced cycle

Autonomous cycle
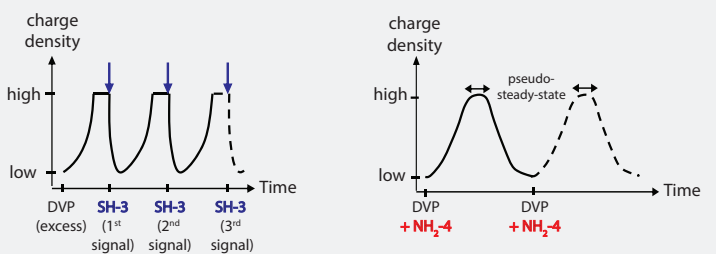

C

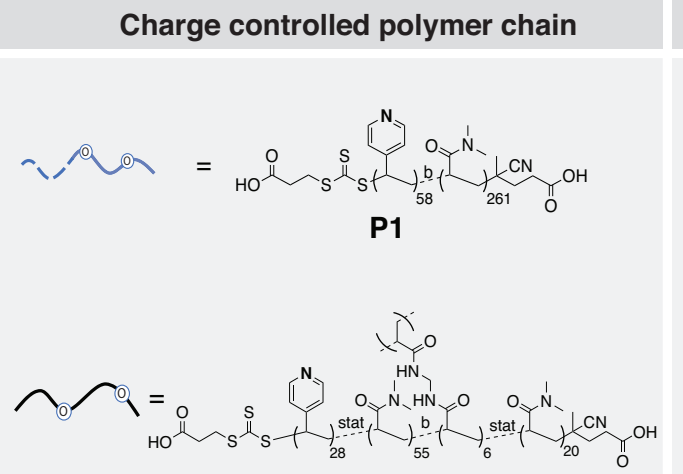

P2

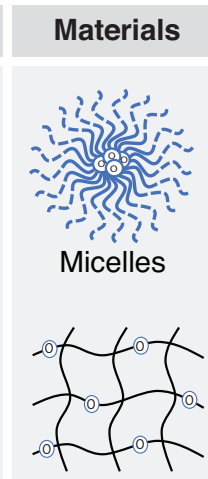

Hydrogel

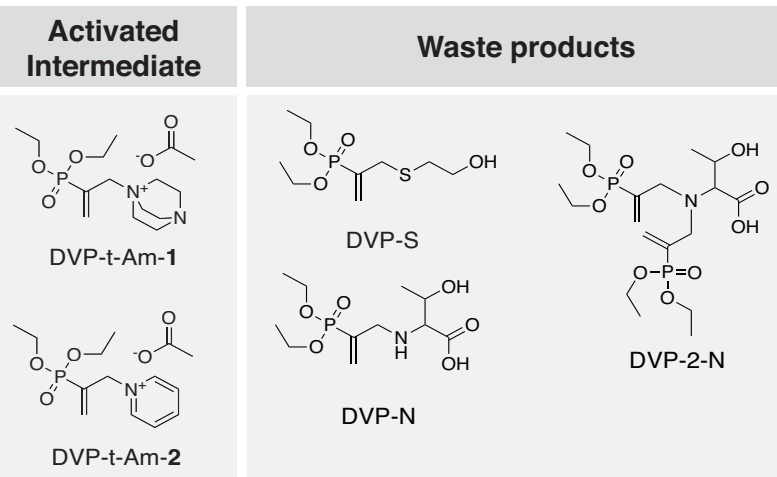

d

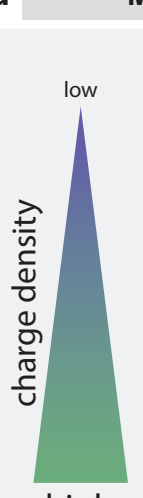

high
Material incorporated CRN

uncharged - polymer chain (P1 or P2)
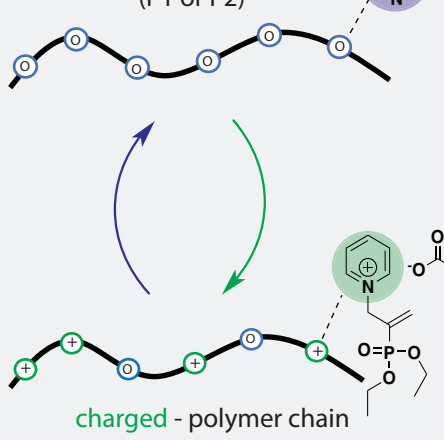

(P1 or P2)

Figure 1: Schematics of the chemical reaction network (CRN), its conditions and components. (a) Generic CRN and CRN strategy used to achieve stimuli-responsive or autonomous material changes. (b) Chemical structure of fuel, nucleophiles and tertiary amines (used in the small molecule model CRN), as well as their activated intermediates and waste products. (c) RAFT synthesised polyamine copolymers (P1, P2) used for the preparation of polymeric materials: (1) micellar dispersions and (2) bulk polymer hydrogels. (d) Temporally programmed charge (de)formation on polyamine-functionalized polymer chains using our CRN strategy and charge density distribution. Specifically, fuelled tertiary amines generate charged quaternary nitrogens (activated intermediate), which in turn creates hydrophilic domains along the polymer backbone. A secondary nucleophilic substitution, results in the regeneration of the uncharged polyamine by substitution of the intermediate (deactivation) towards the waste product. 


\section{Signal-induced and autonomous small-molecule model CRN.}

In the small molecule CRN (Figure 2a), amine substrates t-Am-1 or t-Am-2 react with DVP to generate an intermediary allylammonium ion (DVP-t-Am-1 or DVP-t-Am-2). After fuel activation, reaction with a thiol (SH-3) forms a waste product (DVP-S) and regenerates the amine substrate, thus completing one cycle. Using sub-stoichiometric amounts of amine $(0.2$ eq.) with excess fuel (1.0 eq.) we first converted $t-A m-1$ and $t-A m-2$ to the activated intermediates ( 0.2 eq.), which upon $\mathrm{SH}-3$ addition ( 0.2 eq.) reversed back to the neutral amine. The remaining unreacted fuel ( $\sim 0.8$ eq.) then spontaneously regenerates the activated intermediate, which allows for continuous cycling upon consecutive thiol additions. Using ${ }^{1} \mathrm{H}$ NMR spectroscopy, we confirmed four consecutive reaction cycles by controlled deionization of the activated intermediate with $4 \times \mathrm{SH}-3$ additions (0.2 eq.) at stable $\mathrm{pH}$ conditions (Figure $2 \mathrm{~b} / \mathrm{c}$ ). The blank reaction of DVP with thiol (no tertiary amine) takes $\sim 110$ hours to reach completion (SI, Figure S2.1 \& S2.3), while in the presence of t-Am-1 or t-Am-2 reactivities differ substantially. We attribute these kinetic variations to the difference in nucleophilicity of the employed tertiary amine. ${ }^{42}$

t-Am-1 $\left(N=18.80\right.$ in $\left.\mathrm{CH}_{3} \mathrm{CN}^{43}\right)$ is more nucleophilic compared to t-Am-2 $(N=12.90$ in $\mathrm{CH}_{2} \mathrm{Cl}_{2}{ }^{43}$ ). Hence, it was not surprising that complete conversion of DVP to DVP-t-Am-1 is on average $\sim 9.0 \pm 0.8$ fold faster than that of DVP-t-Am-2 (Figure 2b/c). Similarly, for the progression of the deactivation reaction, DVP-S formed on average $\sim 30 \pm 3.4$ times faster using t-Am-1 than when using t-Am-2 (Figure 2b/c). Although reaction kinetics are amine dependent, in both cases the formation of waste product follows quantitatively after each SH3 addition event (Figure 2b/c), which confirms the absence of unwanted SH-3 side reactivity such as disulfide formation.

Next, we studied systems using the weaker $\mathrm{NH}_{2}-4$ nucleophile in an effort to achieve transient non-equilibrium ionic species formation (Figure 2a). First, we explored the background reaction (no t-Am-2) with a DVP: $\mathrm{NH}_{2}-4$ ratio of 1:4, which reached full conversion after 46 days (SI, Figure S2.2 \& S2.3). Introducing t-Am-2 to the system (t-Am-2:DVP: $\mathrm{NH}_{2}-4=1: 2: 4$ ), we observed the formation of approximately $69 \%$ DVP-t-Am-2 within the first $\sim 32$ h by ${ }^{1} \mathrm{H}$ NMR. 
This was followed by a reaction plateau of $\sim 13 \mathrm{~h}$ (pseudo-steady-state of the activated intermediate, DVP-t-Am-2) and subsequently the recovery of $81 \% \mathrm{t}-\mathrm{Am}-2$ in response to the deactivation reaction (decay of DVP-t-Am-2, Figure 2d). When the network appeared to reach equilibrium ( $\sim 521 \mathrm{~h}$ ), an additional 4.0 eq. of $\mathrm{NH}_{2}-4$ were supplied to the system, which lead to an additional recovery of $12 \% \mathrm{t}-\mathrm{Am}-2$ (93\% total $\mathrm{t}-\mathrm{Am}-2$ recovery) before equilibrium was re-established.

In an effort to tune the amplitude of the activation reaction, we varied the concentration of DVP fuel. Supply of more fuel (4.0 eq.) accelerated the activation reaction with maximum values of $80 \%$ DVP-t-Am-2, as shown in Figure 2e. On the contrary however, the deactivation reaction remained incomplete even with an additional boost of $\mathrm{NH}_{2}-4$, levelling off at $92 \% \mathrm{t}-\mathrm{Am}-2$ recovery. The extra fuel extended the pseudo-steady-state of the activated intermediate (reaction plateau) to $22 \mathrm{~h}$. Evidently, the amount of fuel provides control over the formation of activated intermediate and its reaction plateau. Logically, less fuel (1.0 eq.) slowed down the activation reaction (50\% DVP-t-Am-2 peak formation) and shortened its pseudo-steady-state to $\sim 6 \mathrm{~h}$, while the deactivation reaction remained at a maximum recovery of $94 \% \mathrm{t}-\mathrm{Am}-2$, even after addition of extra $\mathrm{NH}_{2}-4$ (Figure $2 \mathrm{f}$ ). 


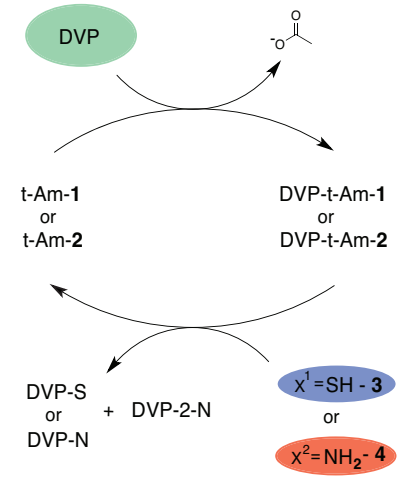

C

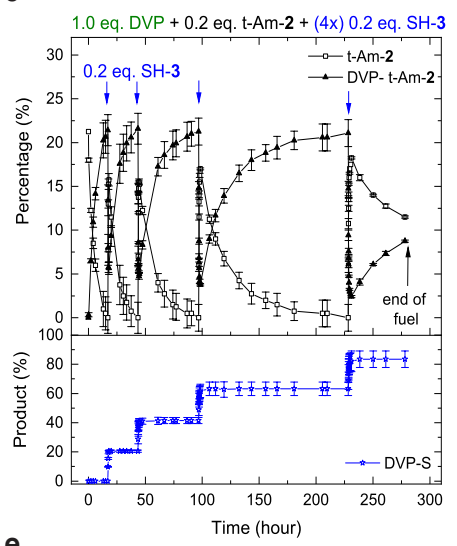

e

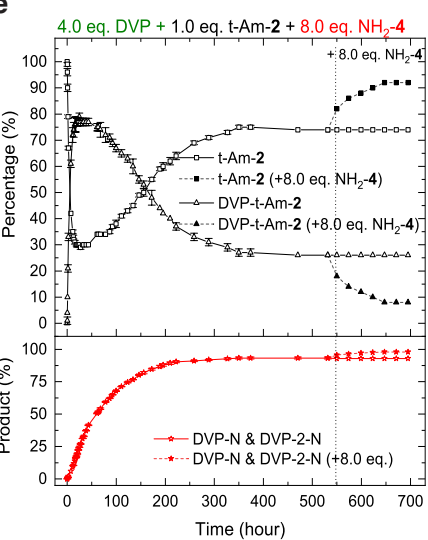

b

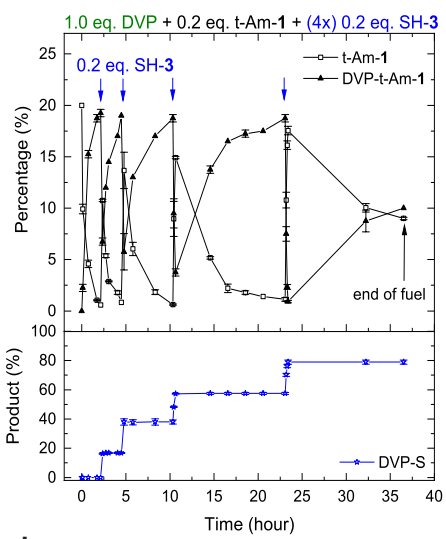

d

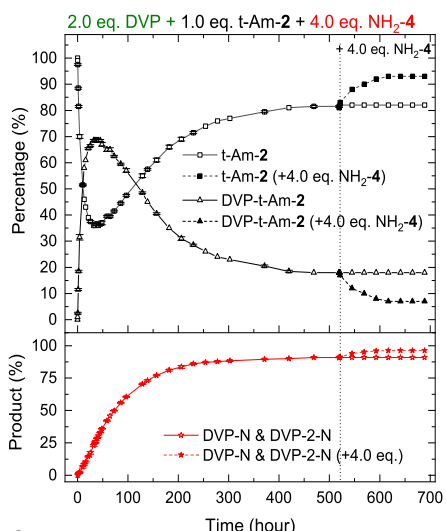

f

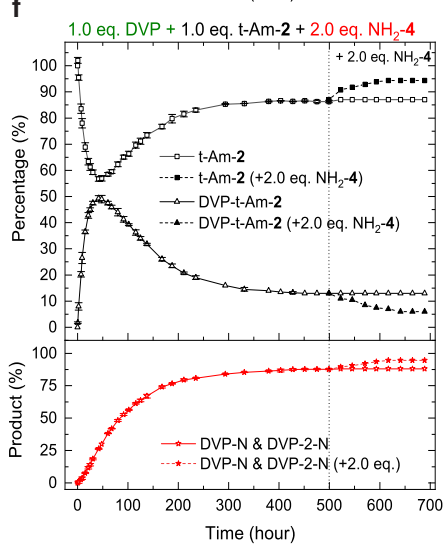

Figure 2: Small molecule CRN model for signal-induced and fuel-driven autonomous cycle. (a) CRN of fuel with tAm-1 (DABCO) or t-Am-2 (pyridine) and SH-3 in signal-induced mode or fuel with t-Am-2 and excess $\mathrm{NH}_{2}-\mathbf{4}$ in fueldriven autonomous mode. Conversion of the reactants was monitored by ${ }^{1} \mathrm{H}$ NMR over time in $\mathrm{D}_{2} \mathrm{O} /$ phosphate buffer $1: 9(\mathrm{pH}=7.4,0.1 \mathrm{M}$ (signal-induced cycle) or $0.5 \mathrm{M}$ (autonomous cycle)) at room temperature. (b) Signal induced cycle (SI, Figure S2.4) with t-Am-1: DVP (42 mM, 1.0 eq.), t-Am-1 (0.2 eq.) and four times addition of SH3 (0.2 eq.). (c) Signal induced cycle (SI, Figure S2.5) with t-Am-2: DVP (42 mM, 1.0 eq.), t-Am-2 (0.2 eq.) and four times addition of SH-3 (0.2 eq.). Autonomous cycle (SI, Figure S2.6 - S2.8) with fuel/nucleophile variations at constant t-Am-2 (8.5 mM, 1.0 eq.) concentration (d): DVP (2.0 eq.) and $\mathrm{NH}_{2}-4$ (4.0 eq.), (e) DVP (4.0 eq.) and $\mathrm{NH}_{2}-$ 4 (8.0 eq.), (f) DVP (1.0 eq.) and $\mathrm{NH}_{2}-4$ (2.0 eq.). (d - f) At observed equilibrium an additional 4.0, 8.0 and 2.0 equivalents of $\mathrm{NH}_{2}-4$ were added to one of the duplicate reaction mixtures respectively. The error bars represent the standard deviation of duplicate measurements. For $(\mathbf{d}, \mathbf{e})$ the product percentages were normalized to 100. Activated intermediates were isolated by exchanging their counterion from acetate to chloride (for full characterisation see SI, Figure S6.7 - S6.16). 


\section{Signal-induced micelle disassembly with programmed cargo release and re-}

uptake.

To program the behaviour of a synthetic material, we combined our CRN with micelle forming block-copolymer P1 (SI, Figure S5.1), which is based on dimethylacrylamide (DMA) as the water-soluble block and 4-vinylpyridine (4VP) as the hydrophobic block (Figure 3a). Upon solubilization of $2.2 \mathrm{mg} / \mathrm{mL} \mathrm{P} 1$ in aqueous-buffer we observed the formation of amphiphilic micelles using dynamic light scattering (DLS) (SI, Figure S3.3, t $=0 \mathrm{~h}$ ) and transmission electron microscopy (TEM). TEM revealed uniform, narrowly dispersed micelles (Figure 3d) with an average diameter ( $D_{\text {TEM }}$ based on a sample population of $n=1202$ in $3.6 \mu m^{2}$ ) of $18 \pm$ $4.4 \mathrm{~nm}$, as determined by statistical image analysis (Figure 3c-top). This value agrees with the Z-averaged hydrodynamic diameter $\left(D_{\mathrm{DLS}}\right)$ obtained from DLS $\left(\mathrm{D}_{\mathrm{DLS}}=52 \mathrm{~nm}\right.$, Figure 3bbottom). The discrepancy between $\mathrm{D}_{\text {TEM }}$ and $\mathrm{D}_{\mathrm{DLS}}$ can be explained by the fact that TEM excludes the length of the hydrophilic DMA chain as a result of dry sample measurements. ${ }^{44}$ Having established the formation of micellar dispersions, we first conducted signal-induced micellar (de)formation experiments with excess fuel (3.2 eq. DVP), P1 (1.0 eq. 4VP) and consecutive $(4 \mathrm{x})$ signal additions (1.0 eq. SH-3) (Figure 3a).

Upon fuelling the micellar solution, the DLS light scatter intensity (scatter count, Figure 3bmiddle) dropped rapidly with a $12 \pm 0.7$ fold reduction (from 8.1 to $0.6 \mathrm{Mcps}$ ) in the first $10 \mathrm{~h}$ reaching its minimum at $\mathrm{t}=105 \mathrm{~h}(0.3 \mathrm{Mcps})$. Simultaneously, ${ }^{1} \mathrm{H}$ NMR measurements showed a $28 \%(t=10 \mathrm{~h})$ to $74 \%(t=105 \mathrm{~h})$ conversion towards the charged activated intermediate (Figure 3b-top). This observation indicates that partial ionization of the micellar core by DVP $(28 \%)$ is sufficient to cause a significant switch in the hydrophilicity of the core, leading to micelle disassembly. Hereafter, the disassembled state equilibrium was slowly reached (up to 105 hours), due to the decreasing presence of neutral polyamine units. At the same time, amine quaternization on the polymer becomes increasingly dominated by charge repulsion effects $^{45}$, which explains the incomplete conversion of DVP $(74 \pm 4.5 \%)$. Micellar solubilization was further confirmed by the measured decrease of the $D_{D L S}$ from $52 \pm 0.4$ to $15 \pm 3.2 \mathrm{~nm}$ 
during the first 105 hours (Figure 3b-bottom), which is consistent with TEM image analysis $\left(D_{\text {TEM }}=13 \pm 3.4 \mathrm{~nm}\right.$ at $\mathrm{t}=105 \mathrm{~h}$, Figure 3c-middle $)$. In addition, the number of residual micellar structures (Figure 3d) decreased substantially $(n=61$ for an identical image area compared to $\mathrm{n}=1202$ at the start).

To complete the cycle, we initiated the deactivation reaction after 105 hours by addition of signal SH-3 (1.0 eq.). We observed a prompt response in ${ }^{1} \mathrm{H}$ NMR and DLS measurements by rapid formation of waste product DVP-S (93 $\pm 8.5 \%$ in $1 \mathrm{~h}$ ), along with an increase in scatter count $(7.2 \pm 0.7$ Mcps $)$ and Z-averaged diameter $\left(D_{\mathrm{DLS}}=41 \pm 2.5 \mathrm{~nm}\right)$ to near starting values (Figure $3 b$ ). Importantly, the re-assembled state (at $t \sim 106 \mathrm{~h}$ ) is not at equilibrium, as unreacted excess fuel ( 2.2 eq.) spontaneously regenerates the quaternized polyamine units. Subsequently, the cycle starts again, until achieving maximum species ionization leading to micelle disassembly. After confirming three additional consecutive cycles, TEM image analysis (Figure 3c-bottom) showed the re-occurrence of micellar structures (Figure 3d) with an average diameter of $16 \pm 3.5 \mathrm{~nm}$ at large population size $\left(\mathrm{n}=1365 \mathrm{in} 3.6 \mu \mathrm{m}^{2}\right)$. However, we also observed that the recovered micellar diameter decreased slightly with each cycle, as seen in TEM (from 18 to $16 \mathrm{~nm}$ ) and DLS (from 51 to $36 \mathrm{~nm}$ ). Such changes may be caused by the increasing accumulation of waste product with each cycle, which is known to limit cycle efficiency. ${ }^{15,46}$

After time-programming micellar (dis)assembly states using $\mathrm{SH}$-signals, we investigated if similar behaviour could be achieved with loaded micelles and whether it is possible to release and re-uptake molecular cargo. To achieve this, micelles were loaded with Nile Red (NR) dye ${ }^{47}$ as model cargo. NR is a solvatochromic dye which exhibits strong fluorescence in hydrophobic environments, while in water its fluorescence is quenched ${ }^{48}$.

We exposed NR-loaded micelles to fuel (3.2 eq. DVP vs. vinyl pyridine) and monitored their fluorescence. During the first 10 hours after fuelling, the fluorescence decreased by $77 \pm 0.3 \%$, ultimately reaching a steady $95 \pm 0.1 \%$ reduction in fluorescence after $52 \mathrm{~h}$ (Figure 3e). Upon SH-3 (1.0 eq.) signal addition, the fluorescence increases promptly, reaching a value close to its original intensity, which corresponds to $87 \pm 0.2 \% \mathrm{NR}$ re-uptake within $4 \mathrm{~h}$ ( $\mathrm{t}=56 \mathrm{~h}$ ). The 
transient increase in fluorescence is attributed to the re-established hydrophobic core unit and hence re-assembled micellar structures. We conducted three additional signal additions cycling between micelle (dis-)assembly states, which corroborate earlier findings without NR in DLS and ${ }^{1} \mathrm{H}$ NMR under identical conditions. Interestingly, we observed an increasingly less efficient cargo re-uptake after each cycle (from $1^{\text {st }}$ cycle: $87 \%$ to $4^{\text {th }}$ cycle: $51 \%$ ). We hypothesized that this behaviour is related to the increasing waste accumulation inside the micelle upon signalling, as seen in micellar fuelling experiments without NR. 
a

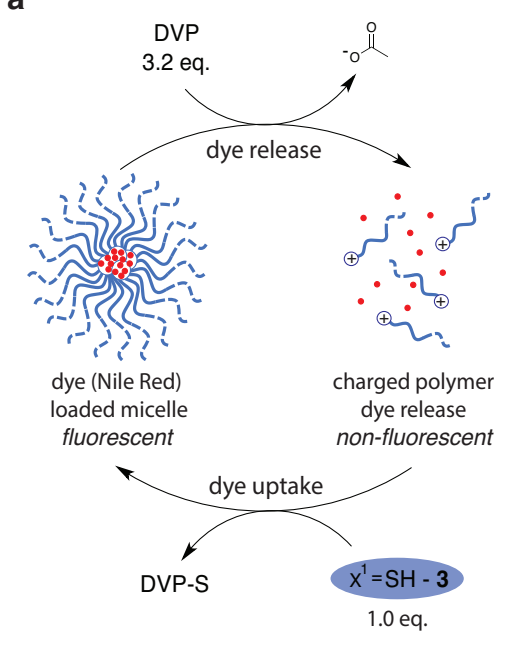

d

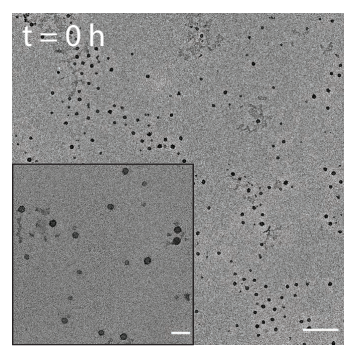

b

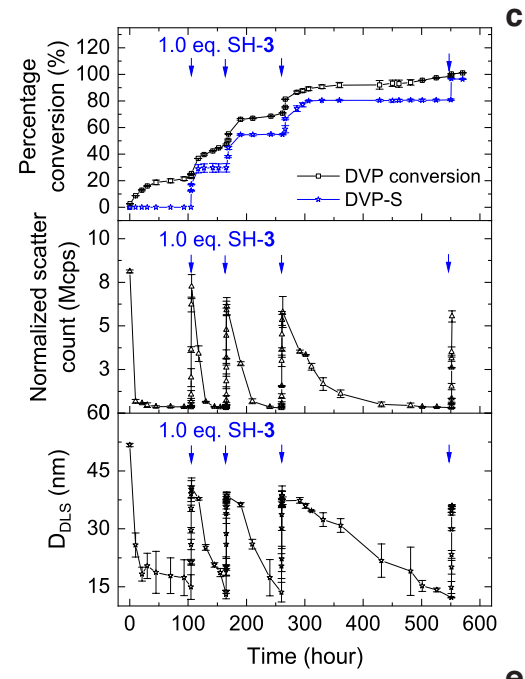

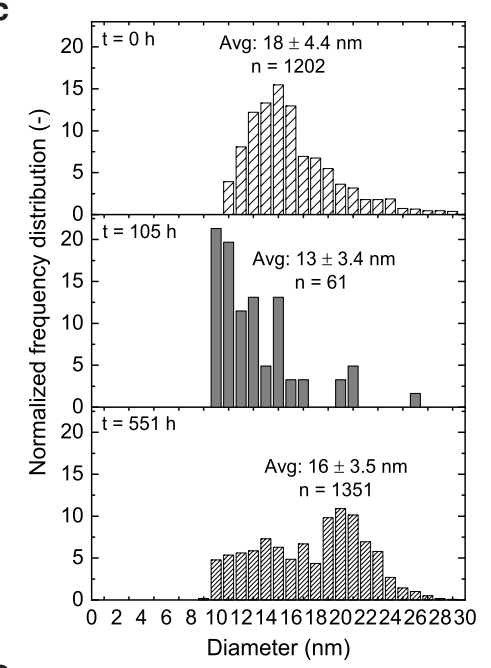

e

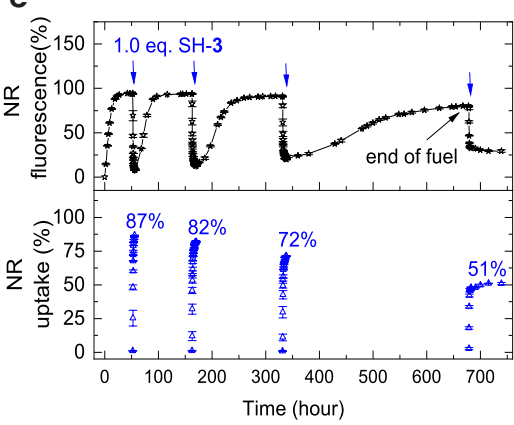

Figure 3: Signal-induced micellar (de)formation reaction network in the absence and presence of Nile Red (NR). (a) Micellar dispersions (2.2 mg/mL P1) were fuelled with DVP (13 mM, 3.2 eq.) and sequential additions of SH-3 (1.0 eq.) as signal (blue arrow) in $1.0 \mathrm{~mL}$ phosphate buffer $\left(0.1 \mathrm{M}, \mathrm{pH}=7.4\right.$ and $10 \% \mathrm{D}_{2} \mathrm{O}$ ) (SI, Figure S3.1). Micellar dispersion in (e) contained $15.5 \pm 0.1 \mu \mathrm{g} \mathrm{NR} / \mathrm{mg}$ of polymer (encapsulation efficiency of $39 \%$ ), while micellar dispersions in (b), (c) and (d) were without NR. (b) Stack graph: (top) DVP conversion and DVP-S formation observed by ${ }^{1} \mathrm{H}$ NMR. (middle) Corresponding DLS measured normalized scatter count (Mcps). (bottom) Corresponding DLS measured Z-averaged diameter ( $D_{\mathrm{DLS}}$ in $\mathrm{nm}$ ). (c) Normalized frequency distribution based on TEM image analysis for micellar (de)formation reaction network. At $t=0 \mathrm{~h}$ : initial micelle formation. At $t=105 \mathrm{~h}$ : disassembled micellar state upon fuel exposure (DVP was added shortly after $t=0 \mathrm{~h}$ ). At $t=551 \mathrm{~h}$ : signal induced micelle (re)assembled state after four sequential cycles and complete consumption of fuel. (d) TEM images of signal-induced micellar (de)formation reaction network (Scale bar: $200 \mathrm{~nm}$, insert: $50 \mathrm{~nm}$ ). (left) At t $=0 \mathrm{~h}$ : with no fuel/ signal. (middle) At $t=105 \mathrm{~h}$ : deformed micelles upon fuel exposure. (right) At $t=551 \mathrm{~h}$ : signal induced micelle (re)formation after four sequential cycles. (e) Micellar (de)formation with corresponding dye uptake profile followed by NR fluorescence at an excitation wavelength of $540 \mathrm{~nm}$ and an emission wavelength of $645 \mathrm{~nm}$. The error bars represent the standard deviation of duplicate measurements. Additional DLS and fluorescence data are presented in Figures S3.3 \& S3.5. 


\section{Autonomous micelle (de)formation with programmed cargo release - uptake.}

Having established controllable signal-responsive micellar (de)formation, we further investigated autonomous cycling by concurrent competition between activation and deactivation reactions in the $\mathrm{CRN}$ with an excess of $\mathrm{NH}_{2}-4$. Autonomous fuelling experiments were performed at optimized conditions with $2.2 \mathrm{mg} / \mathrm{mL} \mathrm{P} 1$ (which corresponds to a 4VP concentration of $4.0 \mathrm{mM}$ ), 2.0 eq. DVP and 8.0 eq. $\mathrm{NH}_{2}-4$ in aqueous-buffer (Figure 4a). The micelle disassembly process reaches its apex at $t=\sim 48 \mathrm{~h}$, corresponding to $59 \pm 2.0 \%$ formation of charged pyridine units (activated intermediate) after 1.1 eq. DVP conversion (55.5 $\pm 0.9 \%$ ) and 0.52 eq. of waste product formation ( $26 \pm 0.2 \%$ DVP-N and DVP-2-N) (Figure $4 \mathrm{~b}-$ top). We found that the transition to the disassembled state occurred upon formation of $\sim 60 \%$ of activated intermediate $(t=48 \mathrm{~h})$, as demonstrated by a simultaneous drop in DLS measured scatter count from 11.9 to $0.5 \mathrm{Mcps}$ and $\mathrm{D}_{\mathrm{DLS}}$ from 53 to $23 \mathrm{~nm}$ (Figure $4 \mathrm{~b}$-middle/bottom). Notably, we observe a larger number of remaining micellar structures $(t=48 \mathrm{~h})$ in contrast to the signal-induced micellar (dis)assembly, in parallel with a $D_{\text {TEM }}$ change from $19 \pm 4.5 \mathrm{~nm}$ ( $\mathrm{n}$ $=1065)$ before fuelling $(t=0 h)$ to $15 \pm 4.2 \mathrm{~nm}(\mathrm{n}=91)$ after $48 \mathrm{~h}$ (Figure $4 \mathrm{c} / \mathrm{d})$.

Nevertheless, from 48 to 504 hours the deactivation reaction kinetically outperforms the activation reaction due to continued depletion of fuel reserves (from 55.5 to $\sim 100 \%$ ). This ultimately leads to further accumulation of waste product (from 26 to $95 \%$ ) until equilibrium is reached after approximately 504 hours (Figure 4b-top). During that period, DLS measured scatter count increases from 0.5 to $11.4 \mathrm{Mcps}$, while $\mathrm{D}_{\mathrm{DLS}}$ recovered to $42 \mathrm{~nm}$ (Figure $4 \mathrm{~b}$ middle/bottom). In good agreement with DLS, TEM image analysis also revealed an increase in micellar diameter $\left(D_{\text {TEM }}=17 \pm 2.6 \mathrm{~nm}\right)$ and qualitatively a much larger number of micellar structures could be observed $(n=706)($ Figure $4 c / d)$. As the CRN depletes its fuel reserve, the rate of deactivation becomes higher than the activation rate and thus reverts the charged polyamine units to their uncharged precursor. This leads to increasing hydrophobicity on the polymer backbone, which ultimately results in reassembled micellar structures at equilibrium state. 
Lastly, we addressed autonomous cargo release and re-uptake by conducting NR probed micellar fuelling experiments at conditions identical to the no-cargo micellar experiments (Figure $4 \mathrm{e}$ ). Using the fuel-driven out-of-equilibrium CRN strategy, $83 \pm 0.3 \%$ of cargo was released in 55 hours. Hereafter, fluorescence intensity steadily increased back to nearly the original level, corresponding to $80 \pm 1.0 \%$ cargo re-uptake over the next $\sim 19$ days. This way we achieved autonomous (dis)assembly of micellar-macromolecular structures with timeprogrammed cargo release and re-uptake. 
a

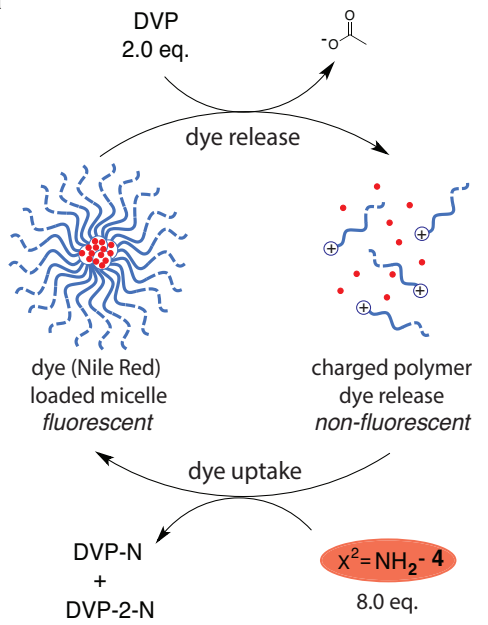

b

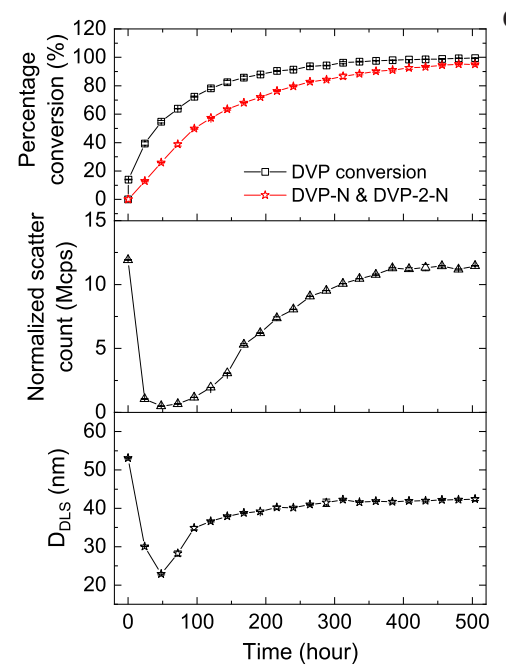

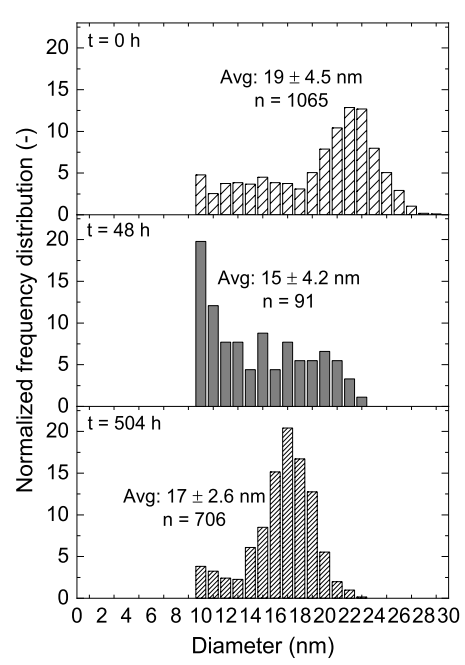

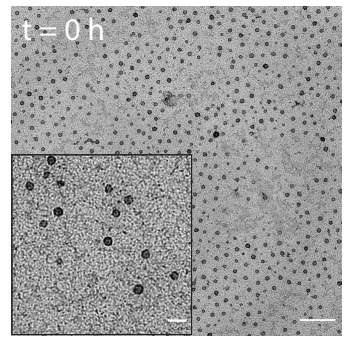

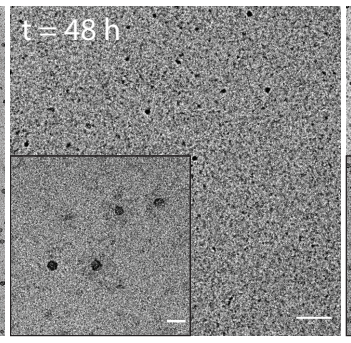

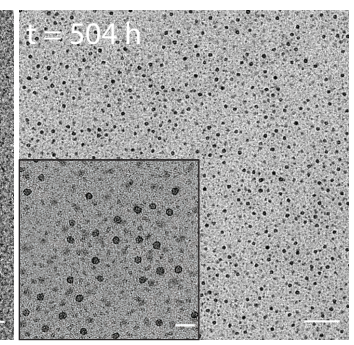

e

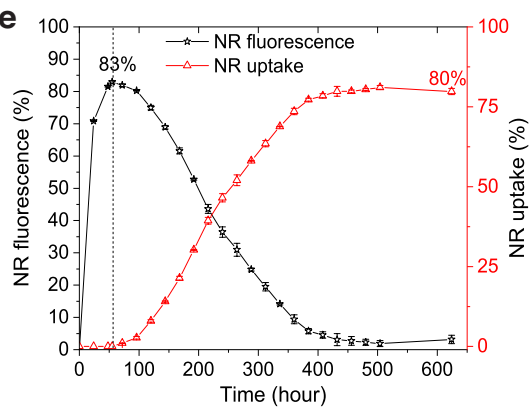

Figure 4: Fuel-driven out-of-equilibrium micellar (de)formation reaction network in the absence and presence of Nile Red (NR). (a) Micellar dispersions (2.2 mg/mL P1) were fuelled with DVP (8.0 mM, 2.0 eq.) and $\mathrm{NH}_{2}-4$ (8.0 eq.) in $1.0 \mathrm{~mL}$ phosphate buffer $\left(0.5 \mathrm{M}, \mathrm{pH}=7.4\right.$ and $\left.10 \% \mathrm{D}_{2} \mathrm{O}\right)$ (SI, Figure S3.2). Micellar dispersion in (e) contained $15.5 \pm 0.1 \mu \mathrm{g} \mathrm{NR} / \mathrm{mg}$ of polymer (encapsulation efficiency of 39\%), while micellar dispersions in (b), (c) and (d) were without NR. (b) Stack graph (top) DVP conversion and DVP-N \& DVP-2-N formation observed by ${ }^{1} \mathrm{H}$ NMR. (middle) Corresponding DLS measured normalized scatter count (Mcps). (bottom) Corresponding DLS measured Z-averaged diameter ( $D_{\mathrm{DLS}}$ in $\mathrm{nm}$ ). (c) Normalized frequency distribution based on TEM image analysis for micellar (de)formation reaction network. At $\mathrm{t}=0 \mathrm{~h}$ : initial micelle formation. At $\mathrm{t}=48 \mathrm{~h}$ : disassembled micellar state upon fuel exposure (DVP was added shortly after $t=0 \mathrm{~h}$ ). At $\mathrm{t}=504 \mathrm{~h}$ : micellar (re)assembled state. (d) TEM images of fuel-driven out-of-equilibrium micellar (de)formation reaction network (Scale bar: $200 \mathrm{~nm}$, insert: $50 \mathrm{~nm}$ ). (left) At $\mathrm{t}=0 \mathrm{~h}$ : with no fuel/ nucleophile. (middle) At $\mathrm{t}=48 \mathrm{~h}$ : micellar deformed state upon fuel/nucleophile exposure. (right) At $\mathrm{t}=504 \mathrm{~h}$ : micellar (re)formation state. (e) Micellar (de)formation with corresponding dye uptake profile followed by NR fluorescence at an excitation wavelength of $540 \mathrm{~nm}$ and an emission wavelength of $645 \mathrm{~nm}$. The error bars represent the standard deviation of duplicate measurements. For (b-top) DVP conversion and product percentages were normalized to 100. Additional DLS and fluorescence data are presented in Figures S3.4 \& S3.5. 


\section{Temporally programmed hydrogel swelling.}

To further demonstrate our CRN strategy, we aimed to achieve reversible expansioncontraction of a polymeric hydrogel network by temporally controlling its water in - and outflux (Figure 5a). We co-polymerized DMA and 4VP to generate a water-soluble statistical copolymer (P2 precursor - SI, Figure S5.2). We then crosslinked this copolymer with bis(acrylamide) to form cube shaped polymer hydrogels (P2) with polyamine concentrations of $97 \mathrm{mg} / \mathrm{mL}$. The resulting gels had a water content of $90 \mathrm{wt} \%$ and dimensions of approximately $1.4 \times 1.2 \times 0.5 \mathrm{~cm}(\mathrm{~L} / \mathrm{W} / \mathrm{H})$. We hypothesized that network expansion can be induced by charge generation upon fuel addition. The increasing concentration of charges in the polymer network will lead to an increase in osmotic pressure in the material which makes the hydrogel swell (expansion) until it is balanced by elastic network forces. ${ }^{41,49}$ In addition, potential repulsive forces between the ionized activated intermediates on the polymer might add to the swelling forces and thus to the expansion of the hydrogel. ${ }^{50}$

To test this hypothesis, polyamine-containing hydrogels were fuelled with DVP (43.2 mM, 1.0 eq. vs. vinyl pyridine). Successful material expansion (Figure 5b) was observed with a maximum of $2010 \pm 158 \%$ increase in swelling percentage (S\%) $96 \mathrm{~h}$ after fuelling (Figure $5 \mathrm{~d}$ blue), while control hydrogels remained at equilibrium weight (Figure $5 \mathrm{~d}$-grey). This drastic increase in volume was further confirmed visually by comparing fuelled hydrogels to their nonfuelled counterparts (Figure 5b). Next, we investigated the reversibility of the system by addition of SH-3 (1.0 eq.). The nucleophilic triggering of the system resulted in hydrogel contraction to near starting values $96 \mathrm{~h}$ after signal introduction ( $\mathrm{S} \%=399 \pm 314 \%)$. To demonstrate the repeatability of the system, signal-responsive (de)swelling was repeated by re-fuelling $(\mathrm{S} \%=1951 \pm 255 \%)$ and subsequent $\mathrm{SH}-3$ signalling $(\mathrm{S} \%=101 \pm 159 \%)$ to temporally program the materials swelling behaviour (Figure 5b/d-blue). Turning our attention to kinetics, we were surprised that swelling proceeds at a similar time-scale as de-swelling ( $t$ $\sim 96 \mathrm{~h}$ ). We suggest here, that the large hydrogel size limits $\mathrm{SH}-3$ diffusion into the material. Another potential factor, which affects the diffusive influx of $\mathrm{SH}-3$, is the convective counterforce of water out-flux with decreasing ionization. 
Next, we explored transient hydrogel swelling by exposing equilibrium-swollen gels to DVP (13.2 mM, 2.1 eq. vs. vinyl pyridine) and excess $\mathrm{NH}_{2}-4$ (8.0 eq.) (Figure 5a). We observed temporary swelling of the polymeric hydrogel network with clear swelling maxima around $t=$ $168 \mathrm{~h}(\mathrm{~S} \%=1636 \pm 222 \%)$, followed by de-swelling which approached starting values (S\% = $88 \pm 119 \%$ ) after 504 hours (Figure 5 c/d-red). This transient swelling study on synthetic hydrogel materials in aqueous media has shown a robust material response and thus the versatility of using the programmed solvent-material interaction strategy for autonomous cycling. 
a

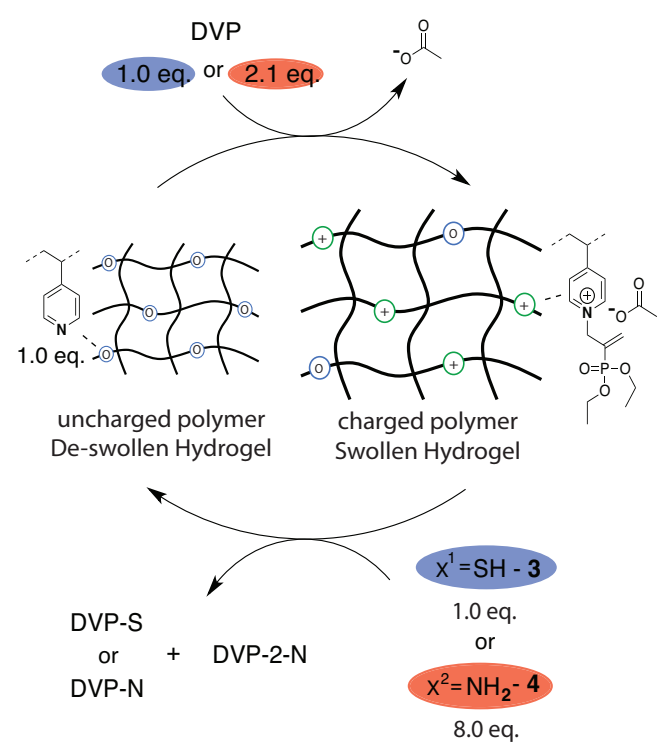

b d

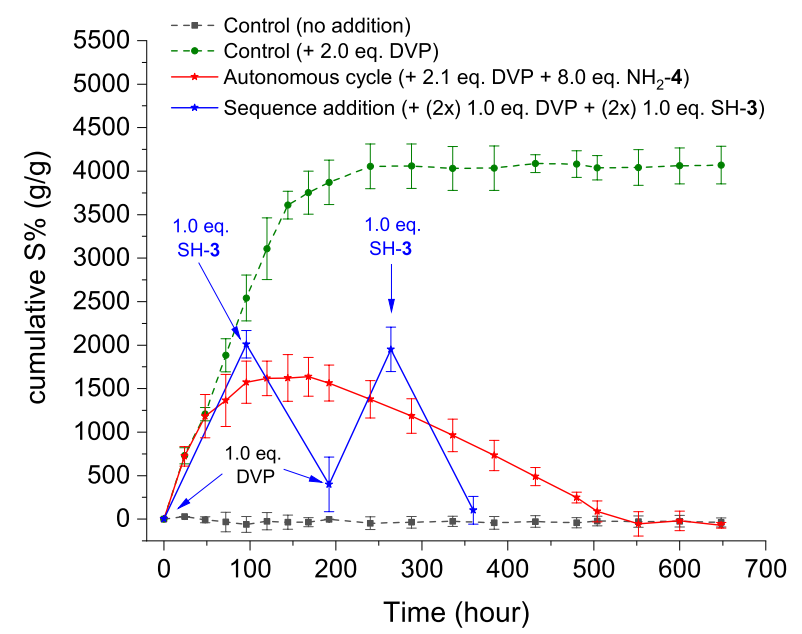

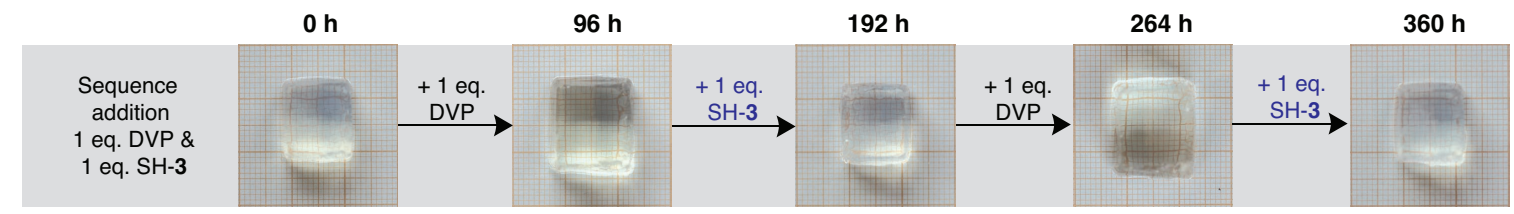

C

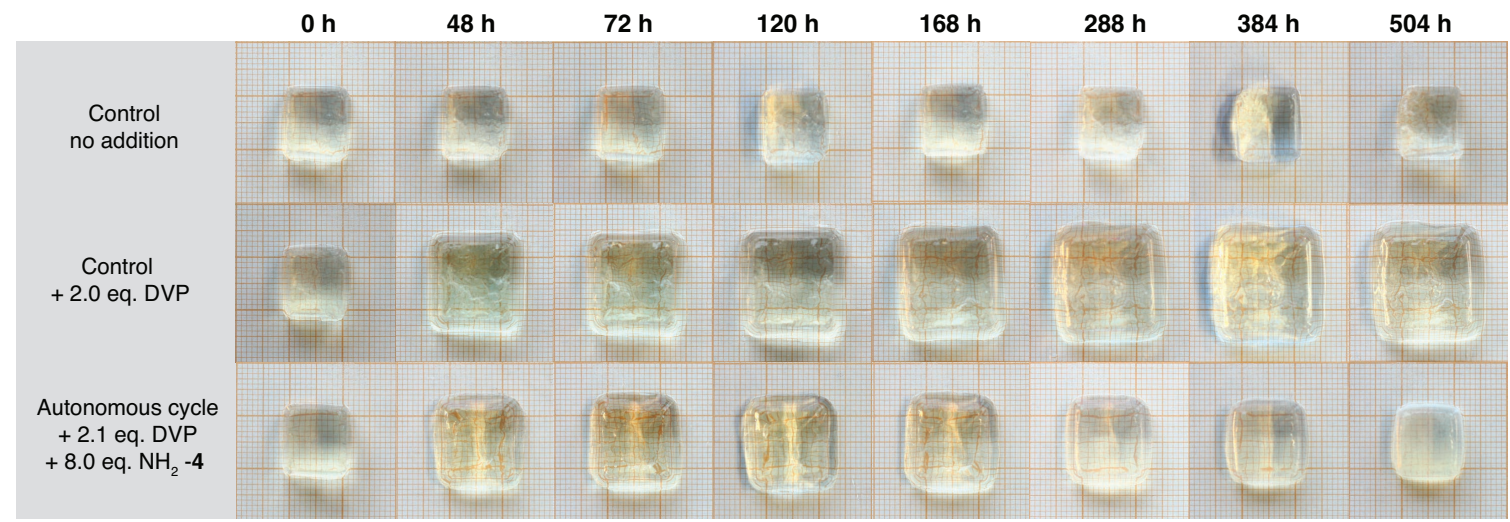

Figure 5: Temporally programmed hydrogel swelling and de-swelling. (a) Hydrogel (de)swelling using signalresponsive or autonomous CRN strategy. The transition from un-swollen to swollen state was monitored visually by time-lapse photographs on millimetre paper and via hydrogel weight measurements (run in triplet) over time in phosphate buffer $(\mathrm{pH}=7.4,0.1 \mathrm{M})$ at room temperature. (b) Time-lapse photographs of signal-responsive hydrogels without solution. (c) Time-lapse photographs of fuel-driven out-of-equilibrium cycle hydrogels without solution in comparison to control hydrogels. (d) Cumulative swelling\% (S\%) for temporally programmed signalresponsive (blue) and autonomous (red) hydrogel (de)swelling over time. Control hydrogel measurements without reactants (grey) and with 2.0 eq. DVP (green). The error bars represent the standard deviation. Additional hydrogel time-lapse photographs are shown in Figure S4.1 - S4.3. 


\section{Conclusion}

In this work, we have introduced a new CRN which operates through successive nucleophilic substitutions on electron deficient allyl acetates (fuel). By first combining a tertiary nitrogen species with the fuel, a cationic quaternary nitrogen intermediate can be formed. This intermediate is stable in $\mathrm{pH} 7.4$ aqueous solutions until undergoing a second nucleophilic substitution with nucleophiles such as a thiol or primary amine. This process regenerates the starting neutral tertiary amine species, along with the formation of a waste product. Unlike most chemically fuelled non-enzymatic CRNs, the deactivation reaction can be controlled at constant $\mathrm{pH}$ by judicious choice of nucleophile. With strong nucleophiles such as 2mercaptoethanol we were able to achieve signal-induced cycling between charge states with excess fuel (DVP) and sequential additions of nucleophile. By switching to weaker nucleophiles such as threonine, both fuel and nucleophile can be introduced simultaneously to yield fuel-driven out-of-equilibrium or autonomous cycling. By incorporating the tertiary nitrogen species into polymeric scaffolds, cycling between neutral and cationic amine species yields transitions between collapsed, hydrophobic polymer chains and solvated, hydrophilic polymer chains. In micellar dispersions, this allowed for the programmed release and reuptake over time of a solvatochromic dye, acting as a model cargo. In bulk polymer hydrogels we used the $\mathrm{CRN}$ to control water influx, allowing us to control hydrogel swelling behaviour by fuel and nucleophile additions. Both material classes could operate under signal-induced and autonomous cycling conditions, leading to different types of behaviour. The principle of temporally programming the behaviour of synthetic materials, shown in this work, is applicable not only to constructing out-of-equilibrium synthetic structures but also as advanced strategy for controlled molecular cargo delivery. We further anticipate that this concept will contribute to the development of next generation soft materials, where signal or time-programmed control over charge density allows for interactive and adaptive material properties, such as stiffening, adhesion or motion. 


\section{Acknowledgement}

The authors acknowledge financial support by the European Research Council (ERC

Consolidator Grant 726381). We thank Dr. G. A. Filonenko for help with the distillation setup, NMR sample preparation and valuable discussions.

\section{References}

1. Walther, A. From responsive to adaptive and interactive materials and materials systems: A roadmap. Adv. Mater. 32, 1905111 (2020).

2. Boekhoven, J. \& Stupp, S. I. 25th anniversary article: supramolecular materials for regenerative medicine. Adv. Mater. 26, 1642-1659 (2014).

3. Hirst, A. R., Escuder, B., Miravet, J. F. \& Smith, D. K. High-tech applications of self-assembling supramolecular nanostructured gel-phase materials: from regenerative medicine to electronic devices. Angew. Chemie Int. Ed. 47, 8002-8018 (2008).

4. Grzybowski, B. A., Fitzner, K., Paczesny, J. \& Granick, S. From dynamic self-assembly to networked chemical systems. Chem. Soc. Rev. 46, 5647-5678 (2017).

5. Boekhoven, J., Hendriksen, W. E., Koper, G. J. M., Eelkema, R. \& van Esch, J. H. Transient assembly of active materials fueled by a chemical reaction. Science (80-. ). 349, 1075-1079 (2015).

6. Karsenti, E. Self-organization in cell biology: a brief history. Nat. Rev. Mol. cell Biol. 9, 255-262 (2008).

7. Leng, Z., Peng, F. \& Hao, X. Chemical-Fuel-Driven Assembly in Macromolecular Science: Recent Advances and Challenges. Chempluschem 85, 1190-1199 (2020).

8. van Rossum, S. A. P., Tena-Solsona, M., van Esch, J. H., Eelkema, R. \& Boekhoven, J. Dissipative outof-equilibrium assembly of man-made supramolecular materials. Chem. Soc. Rev. 46, 5519-5535 (2017).

9. Boekhoven, J. et al. Dissipative self-assembly of a molecular gelator by using a chemical fuel. Angew. Chemie Int. Ed. 122, 4935-4938 (2010).

10. Heuser, T., Weyandt, E. \& Walther, A. Biocatalytic Feedback-Driven Temporal Programming of SelfRegulating Peptide Hydrogels. Angew. Chemie - Int. Ed. 54, 13258-13262 (2015).

11. Panja, S., Patterson, C. \& Adams, D. J. Temporally-Programmed Transient Supramolecular Gels. Macromol. Rapid Commun. 40, 1900251 (2019).

12. Heinen, L. \& Walther, A. Temporal control of i-motif switch lifetimes for autonomous operation of transient DNA nanostructures. Chem. Sci. 8, 4100-4107 (2017).

13. Poros-Tarcali, E. \& Perez-Mercader, J. Concurrent self-regulated autonomous synthesis and 
functionalization of $\mathrm{pH}-$-responsive giant vesicles by a chemical $\mathrm{pH}$ oscillator. Soft Matter 17, 4011-4018 (2021).

14. Wang, G. \& Liu, S. Strategies to Construct a Chemical-Fuel-Driven Self-Assembly. ChemSystemsChem 2, e1900046 (2020).

15. Heckel, J., Loescher, S., Mathers, R. T. \& Walther, A. Chemically Fueled Volume Phase Transition of Polyacid Microgels. Angew. Chemie - Int. Ed. 60, 7117-7125 (2021).

16. Grötsch, R. K. et al. Pathway dependence in the fuel-driven dissipative self-assembly of nanoparticles. J. Am. Chem. Soc. 141, 9872-9878 (2019).

17. Cheng, G. \& Perez-Mercader, J. Dissipative self-assembly of dynamic multicompartmentalized microsystems with light-responsive behaviors. Chem 6, 1160-1171 (2020).

18. Balkenende, D. W. R., Monnier, C. A., Fiore, G. L. \& Weder, C. Optically responsive supramolecular polymer glasses. Nat. Commun. 7, 1-9 (2016).

19. Pappas, C. G. et al. Transient supramolecular reconfiguration of peptide nanostructures using ultrasound. Mater. Horizons 2, 198-202 (2015).

20. Ikegami, T., Kageyama, Y., Obara, K. \& Takeda, S. Dissipative and Autonomous Square-Wave SelfOscillation of a Macroscopic Hybrid Self-Assembly under Continuous Light Irradiation. Angew. Chemie Int. Ed. 128, 8379-8383 (2016).

21. Tena-Solsona, M. et al. Non-equilibrium dissipative supramolecular materials with a tunable lifetime. Nat. Commun. 8, 1-8 (2017).

22. Zhang, B. et al. Chemically fueled covalent crosslinking of polymer materials. Chem. Commun. 55, 2086-2089 (2019).

23. Kariyawasam, L. S., Hossain, M. M. \& Hartley, C. S. The Transient Covalent Bond in Abiotic Nonequilibrium Systems. Angew. Chemie - Int. Ed. 60, 12648-12658 (2021).

24. Wang, G. et al. The fabrication of a supra-amphiphile for dissipative self-assembly. Chem. Sci. 7, 11511155 (2016).

25. Masuda, T. et al. Control of swelling-deswelling behavior of a self-oscillating gel by designing the chemical structure. RSC Adv. 5, 5781-5787 (2015).

26. Wojciechowski, J. P., Martin, A. D. \& Thordarson, P. Kinetically controlled lifetimes in redox-responsive transient supramolecular hydrogels. J. Am. Chem. Soc. 140, 2869-2874 (2018).

27. Wuthrich, K., Weckhuysen, B., Rongy, L. \& De Wit, A. Computational Modeling: From Chemistry To Materials To Biology-Proceedings Of The 25th Solvay Conference On Chemistry. (World Scientific, 2020).

28. Reddi, R. N. et al. Tunable Methacrylamides for Covalent Ligand Directed Release Chemistry. J. Am. Chem. Soc. 143, 4979-4992 (2021). 
29. Zhuang, J. et al. A programmable chemical switch based on triggerable Michael acceptors. Chem. Sci. 11, 2103-2111 (2020).

30. Baidya, M., Remennikov, G. Y., Mayer, P. \& Mayr, H. SN2'versus SN2 Reactivity: Control of Regioselectivity in Conversions of Baylis-Hillman Adducts. Chem. Eur. J. 16, 1365-1371 (2010).

31. Kim, J. N., Lee, H. J., Lee, K. Y. \& Gong, J. H. Regioselective allylic amination of the Baylis-Hillman adducts: An easy and practical access to the Baylis-Hillman adducts of N-tosylimines. Synlett 2002, 173175 (2002).

32. Calcatelli, A., Cherubini-Celli, A., Carletti, E. \& Companyó, X. Unconventional Transformations of MoritaBaylis-Hillman Adducts. Synthesis (Stuttg). 52, 2922-2939 (2020).

33. Chen, G.-Y., Zhong, F. \& Lu, Y. Highly Enantioselective and Regioselective Substitution of MoritaBaylis-Hillman Carbonates with Nitroalkanes. Org. Lett. 13, 6070-6073 (2011).

34. Seingeot, A., Charmasson, Y., Attolini, M. \& Maffei, M. Organocatalyzed synthesis of functionalized vinylphosphonates in water. Heteroat. Chem. 28, e21352 (2017).

35. Wells, P. R. Linear Free Energy Relationships. Chem. Rev. 63, 171-219 (1963).

36. Liu, X.-L., Niu, L.-Y., Chen, Y.-Z., Yang, Y. \& Yang, Q.-Z. A ratiometric fluorescent probe based on monochlorinated BODIPY for the discrimination of thiophenols over aliphatic thiols in water samples and in living cells. Sensors Actuators B Chem. 252, 470-476 (2017).

37. Brotzel, F. \& Mayr, H. Nucleophilicities of amino acids and peptides. Org. Biomol. Chem. 5, 3814-3820 (2007).

38. Henchoz, Y. et al. Rapid determination of $\mathrm{p} \mathrm{K}$ a values of 20 amino acids by CZE with UV and capacitively coupled contactless conductivity detections. Anal. Bioanal. Chem. 389, 1869-1878 (2007).

39. Moad, G., Rizzardo, E. \& Thang, S. H. Living radical polymerization by the RAFT process-a third update. Aust. J. Chem. 65, 985-1076 (2012).

40. Satoh, M., Yoda, E., Hayashi, T. \& Komiyama, J. Potentiometric titration of poly (vinylpyridines) and hydrophobic interaction in the counterion binding. Macromolecules 22, 1808-1812 (1989).

41. De, S. K. et al. Equilibrium swelling and kinetics of $\mathrm{pH}$-responsive hydrogels: Models, experiments, and simulations. J. Microelectromechanical Syst. 11, 544-555 (2002).

42. Shi, Y., Humphrey, G., Maligres, P. E., Reamer, R. A. \& Williams, J. M. Highly Regioselective DABCOCatalyzed Nucleophilic Aromatic Substitution (SNAr) Reaction of Methyl 2, 6-Dichloronicotinate with Phenols. Adv. Synth. Catal. 348, 309-312 (2006).

43. Baidya, M. \& Mayr, H. Nucleophilicities and carbon basicities of DBU and DBN. Chem. Commun. 17921794 (2008).

44. Yan, Q. \& Zhao, Y. CO2-stimulated diversiform deformations of polymer assemblies. J. Am. Chem. Soc. 135, 16300-16303 (2013). 
45. Lemmers, M. et al. The influence of charge ratio on transient networks of polyelectrolyte complex micelles. Soft Matter 8, 104-117 (2012).

46. Pappas, C. G., Sasselli, I. R. \& Ulijn, R. V. Biocatalytic Pathway Selection in Transient Tripeptide Nanostructures. Angew. Chemie - Int. Ed. 54, 8119-8123 (2015).

47. Gupta, M. K., Meyer, T. A., Nelson, C. E. \& Duvall, C. L. Poly (PS-b-DMA) micelles for reactive oxygen species triggered drug release. J. Control. release 162, 591-598 (2012).

48. Greenspan, P. \& Fowler, S. D. Spectrofluorometric studies of the lipid probe, nile red. J. Lipid Res. 26, $781-789$ (1985).

49. Kennedy, J. W. Principles of Polymer Chemistry. Journal of the American Chemical Society vol. 76 (Cornell University Press, 1954).

50. Lai, F. \& Li, H. Modeling of effect of initial fixed charge density on smart hydrogel response to ionic strength of environmental solution. Soft Matter 6, 311-320 (2010). 